\title{
Antiperiodic Solutions for Quaternion-Valued Shunting Inhibitory Cellular Neural Networks with Distributed Delays and Impulses
}

\author{
Nina Huo and Yongkun $\mathrm{Li}$ (D) \\ Department of Mathematics, Yunnan University, Kunming, Yunnan 650091, China \\ Correspondence should be addressed to Yongkun Li; yklie@ynu.edu.cn
}

Received 5 April 2018; Accepted 26 August 2018; Published 15 October 2018

Academic Editor: Xiaoping Liu

Copyright (c) 2018 Nina Huo and Yongkun Li. This is an open access article distributed under the Creative Commons Attribution License, which permits unrestricted use, distribution, and reproduction in any medium, provided the original work is properly cited.

\begin{abstract}
This paper is concerned with quaternion-valued shunting inhibitory cellular neural networks (QVSICNNs) with distributed delays and impulses. By using a new continuation theorem of the coincidence degree theory, the existence of antiperiodic solutions for QVSICNNs is obtained. By constructing a suitable Lyapunov function, some sufficient conditions are derived to guarantee the global exponential stability of antiperiodic solutions for QVSICNNs. Finally, an example is given to show the feasibility of obtained results.
\end{abstract}

\section{Introduction}

The shunting inhibitory cellular neural networks (SICNNs) $[1,2]$ have found many applications in psychophysics, speech, perception, robotics, adaptive pattern recognition, vision, and image processing. Since all of these applications heavily rely on the dynamics of SICNNs and time delays are unavoidable in a realistic system [3-13], there have been extensive results about the dynamical behaviors of SICNNs with time delays [4-10]. Besides, a wide variety of evolutionary processes which exist universally in nature and many signal transmission processes in neural networks are often subject to abrupt changes at certain moments due to instantaneous perturbations which lead to impulsive effects. Also, the existence of impulses is frequently a source of instability, bifurcation, and chaos for neural networks [11-13]. Therefore, many researchers have investigated various dynamical behaviors of SICNNs with time delays and impulses [12, 13].

On the other hand, quaternion-valued neural networks, which can be seen as a generic extension of complex- valued neural networks (CVNNs) or real-valued neural networks (RVNNs), are much more complicated than CVNNs for their quaternion-valued states, quaternionvalued connection weights, and quaternion-valued activation functions. In the past decades, QVNNs have found many practical applications in aerospace and satellite tracking, processing of polarized waves, image processing, 3D geometrical affine transformation, spatial rotation [14, 15], color night vision [16], and so on. Due to so many practical applications, it is necessary to study the dynamics of QVNNs. At present, only a few of the dynamical behaviors of QVNNs have been studied [17-24]. For example, in $[21,22]$, the global $\mu$-stability criteria for QVNNs were studied, respectively; in [23], based on Mawhin's continuation theorem of coincidence degree theory, the existence of periodic solutions for QVNNs was established; in [24], the multiplicity of periodic solutions for QVNNs was discussed by employing Brouwer's and Leray-Schauder's fixed point theorems. However, to the best of our knowledge, the antiperiodic oscillation of QVVNs with time-varying delays and impulses has not been reported. Since the 
existence and stability of antiperiodic solutions are an important topic in nonlinear differential equations and the signal transmission process of neural networks can often be described as an antiperiodic process, the antiperiodic oscillation of neural networks have been considered by many authors, see $[13,25-33]$. So, it is necessary to study the antiperiodic solutions for QVNNs.

Motivated by the above, in this paper, we are concerned with the following QVSICNN with distributed delays and impulsive effects:

$$
\left\{\begin{array}{l}
\dot{x}_{p q}(t)=-a_{p q}(t) x_{p q}(t)-\sum_{C_{k l} \in N_{r}(p, q)} C_{p q}^{k l}(t) \int_{0}^{+\infty} K_{p q}(u) x_{p q}(t) f\left(x_{k l}(t-u)\right) d u+T_{p q}(t), \quad t \geq 0, t \neq t_{h}, h \in \mathbb{N} \\
\Delta x_{p q}\left(t_{h}\right)=x_{p q}\left(t_{h}^{+}\right)-x_{p q}\left(t_{h}^{-}\right)=I_{p q h}\left(x_{p q}\left(t_{h}\right)\right), \quad t=t_{h}, p=1, \ldots, m, q=1, \ldots, n,
\end{array}\right.
$$

where $p q \in\{11,12, \ldots, 1 n, \ldots, m 1, m 2, \ldots, m n\}:=\mathscr{B} ; C_{p q}$ denotes the cell at the $(p, q)$ position of the lattice. The $r$ neighborhood $N_{r}(p, q)$ of $C_{p q}$ is given by

$$
N_{r}(p, q)=\left\{C_{p q}: \max (|k-p|,|l-q|) \leq r, p q \in \mathscr{B}\right\} .
$$

$x_{p q} \in \mathbb{Q}$ is the activity of the cell $C_{p q} ; T_{p q}: \mathbb{Q} \rightarrow \mathbb{Q}$ is the external input to $C_{p q}, a_{p q}(t)>0$ represents the passive decay rate of the cell activity, $C_{p q}^{k l}(t) \geq 0$ is the connection or coupling strength of postsynaptic activity of the cell transmitted to the cell $C_{p q}$, and the activity function $f: \mathbb{Q} \rightarrow \mathbb{Q}$ is a continuous function representing the output or firing rate of the cell $C_{p q} ; K_{p q}(t)$ corresponds to the transmission delay kernel; and $\left\{t_{h}, h \in \mathbb{N}\right\}$ is a sequence of real numbers such that $0<t_{1}<t_{2}<\cdots<t_{h} \rightarrow \infty$ as $h \rightarrow \infty$, there exists a $\mu \epsilon$ $\mathbb{N}$ such that $t_{h+\mu}=t_{h}+\omega / 2, I_{p q(h+\mu)}\left(x_{p q}\left(t_{h+\mu}\right)\right)=-I_{p q h}\left(-x_{p q}\right.$ $\left.\left(t_{h}\right)\right), h \in \mathbb{N}$. Without loss of generality, we also assume that $[0, \omega / 2) \cap\left\{t_{h}: h \in \mathbb{N}\right\}=\left\{t_{1}, t_{2}, \ldots, t_{\mu}\right\}$. For convenience, we denote $\bar{g}=\sup _{t \in \mathbb{R}}|g(t)|$ and $\underline{g}=\inf _{t \in \mathbb{R}}|g(t)|$, where $g$ is a bounded function.

The main purpose of this paper is to establish the existence of antiperiodic solutions to (1) by using a new continuation theorem of coincidence degree theory and by constructing a suitable Lyapunov function to obtain the global exponential stability of the antiperiodic solution. The results of this paper are completely new and supplement the previously known results.

Throughout this paper, we assume Hypotheses 1 to 4 .

(H1) Let $x_{p q}=x_{p q}^{R}+i x_{p q}^{I}+j x_{p q}^{J}+k x_{p q}^{K} \in \mathbb{Q}$, assume that the activity function $f: \mathbb{Q} \rightarrow \mathbb{Q}$ of (1) can be expressed as

$$
\begin{aligned}
f\left(x_{p q}\right)= & f^{R}\left(x_{p q}^{R}, x_{p q}^{I}, x_{p q}^{J}, x_{p q}^{K}\right)+\operatorname{if}^{I}\left(x_{p q}^{R}, x_{p q}^{I}, x_{p q}^{J}, x_{p q}^{K}\right) \\
& +j f^{J}\left(x_{p q}^{R}, x_{p q}^{I}, x_{p q}^{J}, x_{p q}^{K}\right)+k f^{K}\left(x_{p q}^{R}, x_{p q}^{I}, x_{p q}^{J}, x_{p q}^{K}\right),
\end{aligned}
$$

where $f^{v} \in C\left(\mathbb{R}^{4}, \mathbb{R}\right), \quad v \in\{R, I, J, K\}:=E ; \quad$ the impulsive operators $I_{p q h}: \mathbb{Q} \rightarrow \mathbb{Q}$ can be expressed as

$$
\begin{aligned}
I_{p q h}\left(x_{p q}\right)= & I_{p q h}^{R}\left(x_{p q}^{R}, x_{p q}^{I}, x_{p q}^{J}, x_{p q}^{K}\right) \\
& +i I_{p q h}^{I}\left(x_{p q}^{R}, x_{p q}^{I}, x_{p q}^{J}, x_{p q}^{K}\right) \\
& +j I_{p q h}^{I}\left(x_{p q}^{R}, x_{p q}^{I}, x_{p q}^{J}, x_{p q}^{K}\right) \\
& +k I_{p q h}^{K}\left(x_{p q}^{R}, x_{p q}^{I}, x_{p q}^{J}, x_{p q}^{K}\right)
\end{aligned}
$$

where $I_{p q h}^{v} \in C\left(\mathbb{R}^{4}, \mathbb{R}\right), v \in E, p q \in \mathscr{B}$, and $h \in \mathbb{N}$, and the external inputs $T_{p q}: \mathbb{R} \rightarrow \mathbb{Q}$ can be expressed as

$$
T_{p q}(t)=T_{p q}^{R}(t)+i T_{p q}^{I}(t)+j T_{p q}^{J}(t)+k T_{p q}^{K}(t),
$$

where $T_{p q}^{v} \in C(\mathbb{R}, \mathbb{R}), v \in E$, and $p q \in \mathscr{B}$.

(H2) Functions $a_{p q} \in C\left((\mathbb{R},(0,+\infty)), C_{p q}^{k l} \in C(\mathbb{R}, \mathbb{R})\right.$, and $T_{p q} \in C(\mathbb{R}, \mathbb{Q})$, where $p q \in \mathscr{B}$, and there exists a constant $\omega>0$ such that

$$
\begin{aligned}
a_{p q}\left(t+\frac{\omega}{2}\right) & =a_{p q}(t), \\
C_{p q}^{k l}\left(t+\frac{\omega}{2}\right) & =C_{p q}^{k l}(t), \\
T_{p q}\left(t+\frac{\omega}{2}\right) & =-T_{p q}(t), \\
t & \in \mathbb{R},
\end{aligned}
$$

and functions $f, I_{p q h} \in C(\mathbb{Q}, \mathbb{Q})$, where $p q \in \mathscr{B}$ and $h \in \mathbb{N}$, satisfying

$$
\begin{gathered}
f(-x)=f(x), \\
I_{p q h}(-x)=-I_{p q h}(x), \\
x \in \mathbb{Q} .
\end{gathered}
$$


(H3) For $p q \in \mathscr{B}, \int_{0}^{+\infty}\left|K_{p q}(u)\right| \mathrm{d} u<+\infty$.

(H4) For $p q \in \mathscr{B}, u^{v}$, and $v^{v} \in \mathbb{R}$, there exist positive constants $L_{f}^{v}, M_{f}^{v}$, and $I$ such that

$$
\begin{aligned}
& \left|f^{v}\left(u^{R}, u^{I}, u^{J}, u^{K}\right)-f^{v}\left(v^{R}, v^{I}, v^{J}, v^{K}\right)\right| \\
& \leq\left|L_{f}^{R}\right| u^{R}-v^{R}\left|+L_{f}^{I}\right| u^{I}-v^{I} \mid \\
& \quad+L_{f}^{J}\left|u^{J}-v^{J}\right|+L_{f}^{K}\left|u^{K}-v^{K}\right|
\end{aligned}
$$

and

$$
\begin{gathered}
\left|f^{v}\left(u^{R}, u^{I}, u^{J}, u^{K}\right)\right| \leq M_{f}^{v}, \\
\left|I_{p q h}^{v}\left(u^{R}, u^{I}, u^{J}, \mathrm{u}^{K}\right)\right| \leq I,
\end{gathered}
$$

where $f^{v}$ and $I_{p q h}^{v}(u)$ and $v \in E$ are mentioned in H1.

We will adopt the following notations:

$$
\begin{aligned}
& T=\max _{p q \in \mathscr{B}}\left\{\sup _{t \in[0, \omega]}\left|T_{p q}^{v}(t)\right|, v \in E\right\}, \\
& a^{-}=\min _{p q \in \mathscr{B}}\left\{\inf _{t \in[0, \omega]} a_{p q}(t)\right\}, \\
& a^{+}=\max _{p q \in \mathscr{B}}\left\{\sup _{t \in[0, \omega]} a_{p q}(t)\right\} .
\end{aligned}
$$

The initial value of ( 1 ) is given by

$$
x_{p q}(s)=\varphi_{p q}(s) \in \mathbb{Q}, \quad s \in(-\infty, 0] \text {, }
$$

where

$$
\begin{aligned}
\varphi_{p q}(s) & =\varphi_{p q}^{R}(s)+i \varphi_{p q}^{I}(s)+j \varphi_{p q}^{J}(s)+k \varphi_{p q}^{K}(s), \\
\varphi_{p q}^{v} & \in B C((-\infty, 0], \mathbb{R}), \\
p q & \in \mathscr{B} .
\end{aligned}
$$

The remaining part of this paper is organized as follows. In Section 3, some definitions are given. In Section 4, we obtain sufficient conditions for the existence of antiperiodic solutions of (1). In Section 5, the global exponential stability of the antiperiodic solution is studied. In Section 6, we give an example to illustrate the feasibility of the obtained results.

\section{Preliminaries}

The quaternion was invented in 1843 by Hamilton [34]. The skew field of the quaternion is denoted by

$$
\mathbb{Q}:=\left\{q=q_{0}+i q_{1}+j q_{2}+k q_{3}\right\}
$$

where $q_{0}, q_{1}, q_{2}$, and $q_{3}$ are real numbers and the elements $i, j$, and $k$ obey Hamilton's multiplication rules:

$$
\begin{aligned}
& i j=-j i=k, \\
& j k=-k j=i, \\
& k i=-i k=j, \\
& i^{2}=j^{2}=k^{2}=i j k=-1 .
\end{aligned}
$$

In order to avoid the difficulty resulting from the noncommutativity of the quaternion multiplication, by Hamilton's rules and H1, we decompose (1) into the following systems:

$$
\begin{aligned}
& \left\{\begin{aligned}
\dot{x}_{p q}^{R}(t)= & -a_{p q}(t) x_{p q}^{R}(t)-\sum_{C_{k l} \in N_{r}(p, q)} C_{p q}^{k l}(t)\left(\int_{0}^{+\infty} K_{p q}(u) f^{R}[t, u, x] d u x_{p q}^{R}(t)-\int_{0}^{+\infty} K_{p q}(u) f^{I}[t, u, x] d u x_{p q}^{I}(t)\right. \\
& \left.-\int_{0}^{+\infty} K_{p q}(u) f^{J}[t, u, x] d u x_{p q}^{J}(t)-\int_{0}^{+\infty} K_{p q}(u) f^{K}[t, u, x] d u x_{p q}^{K}(t)\right)+T_{p q}^{R}(t) \triangleq F_{p q R}(t, x), \quad t \neq t_{h}, h \in \mathbb{N}, \\
\Delta x_{p q}^{R}\left(t_{h}\right)= & I_{p q h}^{R}\left(x_{p q}\left(t_{h}\right)\right), \quad p q \in \mathscr{B}, h \in \mathbb{N}, \\
& \left.+\int_{0}^{+\infty} K_{p q}(u) f^{J}[t, u, x] d u x_{p q}^{K}(t)-\int_{0}^{+\infty} K_{p q}(u) f^{K}[t, u, x] d u x_{p q}^{J}(t)\right)+T_{p q}^{I}(t) \triangleq F_{p q I}(t, x), \quad t \neq t_{h}, h \in \mathbb{N}, \\
\dot{x}_{p q}^{I}(t)= & -a_{p q}(t) x_{p q}^{I}(t)-\sum_{C_{k l} \in N_{r}(p, q)} C_{p q}^{k l}(t)\left(\int_{0}^{+\infty} K_{p q}(u) f^{R}[t, u, x] d u x_{p q}^{I}(t)+\int_{0}^{+\infty} K_{p q}(u) f^{I}[t, u, x] d u x_{p q}^{R}(t)\right. \\
\Delta x_{p q}^{I}\left(t_{h}\right)= & I_{p q h}^{I}\left(x_{p q}\left(t_{h}\right)\right), \quad p q \in \mathscr{B}, h \in \mathbb{N}, \\
& \left.+\int_{0}^{+\infty} K_{p q}(u) f^{J}[t, u, x] d u x_{p q}^{R}(t)+\int_{0}^{+\infty} K_{p q}(u) f^{K}[t, u, x] d u x_{p q}^{I}(t)\right)+T_{p q}^{J}(t) \triangleq F_{p q J}(t, x), \quad t \neq t_{h}, h \in \mathbb{N}, \\
\dot{x}_{p q}^{J}(t)= & -a_{p q}(t) x_{p q}^{J}(t)-\sum_{C_{k l} \in N_{r}(p, q)} C_{p q}^{k l}(t)\left(\int_{0}^{+\infty} K_{p q}(u) f^{R}(t, u, x) d u x_{p q}^{J}(t)-\int_{0}^{+\infty} K_{p q}(u) f^{I}[t, u, x] d u x_{p q}^{K}(u)\right.
\end{aligned}\right. \\
& \left\{\begin{array}{l}
\Delta x_{p q}^{J}\left(t_{h}\right)= \\
I_{p q h}^{J}\left(x_{p q}\left(t_{h}\right)\right), \quad p q \in \mathscr{B}, h \in \mathbb{N},
\end{array}\right.
\end{aligned}
$$




$$
\left\{\begin{aligned}
\dot{x}_{p q}^{K}(t)= & -a_{p q}(t) x_{p q}^{K}(t)-\sum_{C_{k l} \in N_{r}(p, q)} C_{p q}^{k l}(t)\left(\int_{0}^{+\infty} K_{p q}(u) f^{R}[t, u, x] d u x_{p q}^{K}(t)+\int_{0}^{+\infty} K_{p q}(u) f^{I}[t, u, x] d u x_{p q}^{J}(t)\right. \\
& \left.-\int_{0}^{+\infty} K_{p q}(u) f^{J}[t, u, x] d u x_{p q}^{I}(t)+\int_{0}^{+\infty} K_{p q}(u) f^{K}[t, u, x] d u x_{p q}^{R}(t)\right)+T_{p q}^{K}(t) \triangleq F_{p q K}(t, x), \quad t \neq t_{h}, h \in \mathbb{N}, \\
\Delta x_{p q}^{K}\left(t_{h}\right)= & I_{p q h}^{K}\left(x_{p q}\left(t_{h}\right)\right), \quad p q \in \mathscr{B}, h \in \mathbb{N},
\end{aligned}\right.
$$

where $x_{p q}^{R}+i x_{p q}^{I}+j x_{p q}^{J}+k x_{p q}^{K}=x_{p q}, f^{v}[t, u, x] \triangleq f^{v}\left(x_{k l}^{R}(t-u)\right.$, $\left.x_{k l}^{I}(t-u), x_{k l}^{J}(t-u), x_{k l}^{K}(t-u)\right)$, and $I_{p q h}^{v}\left(x_{p q}\left(t_{h}\right)\right) \triangleq I_{p q h}^{v}\left(x_{p q}^{R}\right.$ $\left.\left(t_{h}\right), x_{p q}^{I}\left(t_{h}\right), x_{p q}^{J}\left(t_{h}\right), x_{p q}^{K}\left(t_{h}\right)\right), p q \in \mathscr{B}, h \in \mathbb{N}$, and $v \in E$. That is, (1) can be decomposed as the following realvalued system:

$$
\left\{\begin{array}{l}
\dot{x}_{p q}^{v}(t)=F_{p q v}(t, x), \quad t \neq t_{h}, h \in \mathbb{N}, \\
\Delta x_{p q}^{v}\left(t_{h}\right)=I_{p q h}^{v}\left(x_{p q}\left(t_{h}\right)\right), \quad p q \in \mathscr{B}, h \in \mathbb{N}, v \in E,
\end{array}\right.
$$

with the initial values

$$
x_{p q}^{v}(s)=\varphi_{p q}^{v}(s), \quad s \in(-\infty, 0], h \in \mathbb{N}, p q \in \mathscr{B}, v \in E,
$$

where $\varphi_{p q}^{v}:(-\infty, 0] \rightarrow \mathbb{R}$ is continuous and bounded.

Definition 1. A piecewise continuous function $x=\left(x_{11}^{R}, x_{11}^{I}\right.$, $\left.x_{11}^{J}, x_{11}^{K}, \ldots, x_{m n}^{R}, x_{m n}^{I}, x_{m n}^{J}, x_{m n}^{K}\right)^{T}: \mathbb{R} \rightarrow \mathbb{R}^{4 n m}$ is said to be a solution of (16), if

(i) $x(s)=\varphi(s)$ for $s \in(-\infty, 0]$, where $\varphi=\left(\varphi_{11}^{R}, \varphi_{11}^{I}, \varphi_{11}^{J}\right.$, $\left.\varphi_{11}^{K}, \ldots, \varphi_{m n}^{R}, \varphi_{m n}^{I}, \varphi_{m n}^{J}, \varphi_{m n}^{K}\right)^{T}, \quad \varphi_{p q}^{v} \in C((-\infty, 0], \mathbb{R})$, $v \in E$, and $p q \in \mathscr{B}$;

(i) $x(t)$ satisfies (16) for $t \geq 0$;

(ii) $x(t)$ is continuous everywhere except for some $t_{h}$ and left continuous at $t=t_{h}$, and the right limit $x\left(t_{h}^{+}\right)$ exists for $h \in \mathbb{N}$.

Definition 2. A solution $x$ of system (16) is said to be the $\omega / 2$-antiperiodic solution of (16), if

$$
\left\{\begin{array}{l}
x\left(t+\frac{\omega}{2}\right)=-x(t), \quad t \neq t_{h}, \\
x\left(\left(t_{h}+\frac{\omega}{2}\right)^{+}\right)=-x\left(t_{h}^{+}\right), \quad h \in \mathbb{N} .
\end{array}\right.
$$

Definition 3. Let $x=\left(x_{11}^{R}, x_{11}^{I}, x_{11}^{J}, x_{11}^{K}, \ldots, x_{m n}^{R}, x_{m n}^{I}, x_{m n}^{J}\right.$, $\left.x_{m n}^{K}\right)^{T}$ be a solution of (16) with the initial value $\varphi=$ $\left(\varphi_{11}^{R}, \varphi_{11}^{I}, \varphi_{11}^{J}, \varphi_{11}^{K}, \ldots, \varphi_{m n}^{R}, \varphi_{m n}^{I}, \varphi_{m n}^{J}, \varphi_{m n}^{K}\right)^{T}$ and $y=\left(y_{11}^{R}, y_{11}^{I}\right.$, $\left.y_{11}^{J}, y_{11}^{K}, \ldots, y_{m n}^{R}, y_{m n}^{I}, y_{m n}^{J}, x_{m n}^{K}\right)^{T}$ be an arbitrary solution of
(16) with the initial value $\psi=\left(\psi_{11}^{R}, \psi_{11}^{I}, \psi_{11}^{J}, \psi_{11}^{K}, \ldots, \psi_{m n}^{R}\right.$, $\left.\varphi_{m n}^{I}, \varphi_{m n}^{J}, \varphi_{m n}^{K}\right)^{T}$. If there exist constants $\lambda>0$ and $\mathrm{M}>0$ such that

$$
\|x(t)-y(t)\|_{0} \leq M\|\varphi-\psi\| e^{-\lambda t}, \quad \forall t>0
$$

then the solution $x$ of (16) is said to be globally exponentially stable, where

$$
\begin{aligned}
& \|y(t)-x(t)\|_{0}=\max _{p q \in \mathscr{B}}\left\{\max _{v \in E}\left|y_{p q}^{v}(t)-x_{p q}^{v}(t)\right|\right\}, \\
& \|\varphi-\psi\|=\sum_{p q \in \mathscr{B} s \in(-\infty, 0]} \sup _{s q}\left(\left|\varphi_{p q}^{R}(s)-\psi_{p q}^{R}(s)\right|\right. \\
& +\left|\varphi_{p q}^{I}(s)-\psi_{p q}^{I}(s)\right|+\left|\varphi_{p q}^{J}(s)-\psi_{p q}^{J}(s)\right| \\
& \left.+\left|\varphi_{p q}^{K}(s)-\psi_{p q}^{K}(s)\right|\right) .
\end{aligned}
$$

Remark 1. If $x=\left(x_{11}^{R}, x_{11}^{I}, x_{11}^{J}, x_{11}^{K}, \ldots, x_{m n}^{R}, x_{m n}^{I}, x_{m n}^{J}, x_{m n}^{K}\right)^{T}$ is an $\omega / 2$-antiperiodic solution to (16), then $u=\left(x_{11}, \ldots\right.$, $\left.x_{m n}\right)^{T}$, where $x_{p q}=x_{p q}^{R}+i x_{p q}^{I}+j x_{p q}^{J}+k x_{p q}^{K}, p q \in E$ must be an $\omega / 2$-antiperiodic solution to (1). Thus, the problem of finding an $\omega / 2$-antiperiodic solution for (1) reduces to finding one for the system of (16). For considering the stability of the solution of (1), we just need to consider the stability of the solutions of (16).

\section{The Existence of Antiperiodic Solutions}

In this section, based on a new continuation theorem of coincidence degree theory, we shall study the existence of antiperiodic solutions of (1).

Lemma 1. [35] Let $X$ and $Y$ be Banach spaces, and let $L$ : Dom $L \subset X \rightarrow Y$ be linear and $N: X \rightarrow Y$ be continuous. Assume that $L$ is one-to-one and $K:=L^{-1} N$ is compact. Furthermore, assume that there exists a bounded and open subset $\Omega \subset X$ with $0 \in \Omega$ such that equation $L u=\lambda N u$ has no solutions in $\partial \Omega \cap$ Dom $L$ for any $\lambda \in(0,1)$. Then the problem $L u=N u$ has at least one solution in $\bar{\Omega}$.

Theorem 1. Assume that H1-H4 holds. Furthermore, suppose that

\section{A1. If one has}




$$
\begin{gathered}
\Gamma=\frac{a^{-} \omega\left(1-a^{+} \omega\right)}{1+a^{-} \omega}-\left(M_{f}^{R}+M_{f}^{I}+M_{f}^{J}+M_{f}^{K}\right) \\
\cdot \sum_{C_{k l} \in N_{r}(p, q)} \bar{C}_{p q}^{k l} \int_{0}^{+\infty}\left|K_{p q}(u)\right| d u>0,
\end{gathered}
$$

then (1) has at least one $\omega / 2$-antiperiodic solution.

Proof 1. One has

$$
\begin{aligned}
P C\left(\mathbb{R}, \mathbb{R}^{4 m n}\right)= & \left\{x=\left(x_{11}^{R}, x_{11}^{I}, x_{11}^{J}, x_{11}^{K}, \ldots, x_{m n}^{R},\right.\right. \\
& \left.x_{m n}^{I}, x_{m n}^{J}, x_{m n}^{K}\right)^{T}: \mathbb{R} \rightarrow \mathbb{R}^{4 m n},\left.x\right|_{\left(t_{h}, t_{h+1}\right]} \\
\in & C\left(\left(t_{h}, t_{h+1},\right] \mathbb{R}^{4 n}\right), x\left(t_{h}^{+}\right), \\
& \left.x\left(t_{h}\right) \text { exist and } x\left(t_{h}^{-}\right)=x\left(t_{h}\right), h \in \mathbb{N}\right\} .
\end{aligned}
$$

Set

$$
\begin{aligned}
X= & \left\{x: x=\left(x_{11}^{R}, x_{11}^{I}, x_{11}^{J}, x_{11}^{K}, \ldots, x_{m n}^{R}, x_{m n}^{I}, x_{\mathrm{m} n}^{J}, x_{m n}^{K}\right)^{T}\right. \\
& \left.\in P C\left(\mathbb{R}, \mathbb{R}^{4 m n}\right), x\left(t+\frac{\omega}{2}\right)=-x(t)\right\},
\end{aligned}
$$

and

$$
Y=X \times \mathbb{R}^{4 m n \times \mu} .
$$

Then $X$ is a Banach space with the norm $\|x\|_{X}=$ $\max _{p q \in \mathscr{B}}\left\{\max _{v \in E}\left\{\sup _{t \in \mathbb{R}}\left|x_{p q}^{v}(t)\right|\right\}\right\}$, and $Y$ is also a Banach space with the norm $\|y\|_{Y}=\|x\|_{X}+\|u\|, x \in X, u \in \mathbb{R}^{4 m n \times \mu}$, where $\|\cdot\|$ is any norm of $\mathbb{R}^{4 m n \times \mu}$.

Define a linear operator $L: \operatorname{Dom} L \subset X \rightarrow Y$ by

$$
L x=\left(\dot{x}, \Delta x\left(t_{1}\right), \Delta x\left(t_{2}\right), \ldots, \Delta x\left(t_{\mu}\right)\right),
$$

where $\operatorname{Dom} L=\{x: x \in X, \dot{x} \in X\}$ and a continuous operator $N: X \rightarrow Y$ by

$$
N x=\left(\left(\begin{array}{c}
F_{11 R}(t, x) \\
F_{11 I}(t, x) \\
F_{11 J}(t, x) \\
F_{11 K}(t, x) \\
\vdots \\
F_{m n R}(t, x) \\
F_{m n I}(t, x) \\
F_{m n I}(t, x) \\
F_{m n K}(t, x)
\end{array}\right),\left(\begin{array}{c}
I_{111}^{R}\left(x_{11}\left(t_{1}\right)\right) \\
I_{111}^{I}\left(x_{11}\left(t_{1}\right)\right) \\
I_{111}^{I}\left(x_{11}\left(t_{1}\right)\right) \\
I_{111}^{K}\left(x_{11}\left(t_{1}\right)\right) \\
\vdots \\
I_{m n 1}^{R}\left(x_{m n}\left(t_{1}\right)\right) \\
I_{m n 1}^{I}\left(x_{m n}\left(t_{1}\right)\right) \\
I_{m n 1}^{J}\left(x_{m n}\left(t_{1}\right)\right) \\
I_{m n 1}^{K}\left(x_{m n}\left(t_{1}\right)\right)
\end{array}\right),\left(\begin{array}{c}
I_{112}^{R}\left(x_{11}\left(t_{2}\right)\right) \\
I_{112}^{I}\left(x_{11}\left(t_{2}\right)\right) \\
I_{112}^{I}\left(x_{11}\left(t_{2}\right)\right) \\
I_{112}^{K}\left(x_{11}\left(t_{2}\right)\right) \\
\vdots \\
I_{m n 2}^{R}\left(x_{m n}\left(t_{2}\right)\right) \\
I_{m n 2}^{I}\left(x_{m n}\left(t_{2}\right)\right) \\
I_{m n 2}^{I}\left(x_{m n}\left(t_{2}\right)\right) \\
I_{m n 2}^{K}\left(x_{m n}\left(t_{2}\right)\right)
\end{array}\right), \ldots,\left(\begin{array}{c}
I_{11 \mu}^{R}\left(x_{11}\left(t_{\mu}\right)\right) \\
I_{11 \mu}^{I}\left(x_{11}\left(t_{\mu}\right)\right) \\
I_{11 \mu}^{I}\left(x_{11}\left(t_{\mu}\right)\right) \\
I_{11 \mu}^{K}\left(x_{11}\left(t_{\mu}\right)\right) \\
\vdots \\
I_{m n \mu}^{R}\left(x_{m n}\left(t_{\mu}\right)\right) \\
I_{m n \mu}^{I}\left(x_{m n}\left(t_{\mu}\right)\right) \\
I_{m n \mu}^{I}\left(x_{m n}\left(t_{\mu}\right)\right) \\
I_{m n \mu}^{K}\left(x_{m n}\left(t_{\mu}\right)\right)
\end{array}\right)\right) .
$$

It is easy to see that

$$
\operatorname{ker} L=\{0\} \text {, }
$$

$\operatorname{Im} L=\left\{y=\left(g, d_{1}, \ldots, d_{11}\right)^{T} \in \mathbb{Y}: \int_{0}^{\omega} g(s) d s=0\right\}=\mathbb{Y}$.

Hence, $L$ is reversible. Denote by $L^{-1}: \operatorname{Im} L \rightarrow \operatorname{Dom} L$ the inverse of $L$, one has

$\left(L^{-1} y\right)(t)=\int_{0}^{t} g(s) d s-\frac{1}{2} \int_{0}^{\omega / 2} g(s) d s+\sum_{t_{h}<t} d_{h}-\frac{1}{2} \sum_{h=1}^{u} d_{h}, \quad t \in \mathbb{R}$, where $d_{\mu+h}=d_{h}$ for all $1 \leq h \leq \mu$. Let $K:=L^{-1} N$, by applying the Arzela-Ascoli theorem, we know that $K$ is compact. Corresponding to the operator equation $L x=\lambda N x, \lambda \in(0,1)$, we have

$$
\left\{\begin{array}{l}
\dot{x}_{p q}^{v}(t)=\lambda F_{p q v}(t, x), \quad t \geq 0, t \neq t_{h}, \\
\Delta x_{p q}^{v}\left(t_{h}\right)=\lambda I_{p q h}^{v}\left(x_{p q}\left(t_{h}\right)\right), \quad p q \in \mathscr{B}, h \in \mathbb{N}, v \in E .
\end{array}\right.
$$

Suppose that $x=\left(x_{11}^{R}, x_{11}^{I}, x_{11}^{J}, x_{11}^{K}, \ldots, x_{m n}^{R}, x_{m n}^{I}, x_{m n}^{J}\right.$, $\left.x_{m n}^{K}\right)^{T} \in X$ is a solution of (29) for a certain $\lambda \in(0,1)$, set $t_{0}=t_{0}^{+}=0, t_{2 q+1}=\omega$, then we have 


$$
\begin{array}{rlrl}
\int_{0}^{\omega}\left|\dot{x}_{p q}^{R}(t)\right| d t & =\sum_{k=1}^{2 q+1} \int_{t_{h-1}^{+}}^{t_{h}}\left|\dot{x}_{p q}^{R}(t)\right| d t+\sum_{h=1}^{2 q}\left|I_{p q h}^{R}\left(x_{p q}\left(t_{h}\right)\right)\right| & \int_{0}^{\omega}\left|\dot{x}_{p q}^{v}(t)\right| d t \leq a^{+} \omega\left|x_{p q}^{v}\right|_{\infty}+\omega\left(M_{f}^{R}+M_{f}^{I}+M_{f}^{J}+M_{f}^{K}\right) \\
\leq \int_{0}^{\omega}\left|a_{p q}(t) x_{p q}^{R}(t)\right| d t+\int_{0}^{\omega} \mid \sum_{C_{k l} \in N_{r}(p, q)} C_{p q}^{k l}(t) & \cdot\|x\|_{X} \sum_{C_{k l} \in N_{r}(p, q)} \bar{C}_{p q}^{k l} \int_{0}^{+\infty}\left|K_{p q}(u)\right| d u \\
& +\omega T+2 q I, \quad p q \in \mathscr{B} .
\end{array}
$$$$
\left(\int_{0}^{+\infty} K_{p q}(u) x_{p q}^{R}(t) f^{R}[t, u, x] d u\right.
$$$$
-\int_{0}^{+\infty} K_{p q}(u) x_{p q}^{I}(t) f^{I}[t, u, x] d u
$$$$
-\int_{0}^{+\infty} K_{p q}(u) x_{p q}^{J}(t) f^{J}[t, u, x] d u \mid
$$$$
\left.-\int_{0}^{+\infty} K_{p q}(u) x_{p q}^{K}(t) f^{K}[t, u, x] d u\right) \mid d t
$$$$
+\int_{0}^{+\infty}\left|T_{p q}^{R}(t)\right| d t+\sum_{h=1}^{2 q}\left|I_{p q h}^{R}\left(x_{p q}\left(t_{h}\right)\right)\right|
$$$$
\leq \int_{0}^{\omega}\left|a_{p q}(t) x_{p q}^{R}(t)\right| d t+\int_{0}^{\omega} \sum_{C_{k l} \in N_{r}(p, q)} \bar{C}_{p q}^{k l}
$$$$
\left(\int_{0}^{+\infty}\left|K_{p q}(u)\left\|x_{p q}^{R}(t)\right\| f^{R}[t, u, x]\right| d u\right.
$$$$
+\int_{0}^{+\infty}\left|K_{p q}(u)\right|\left|x_{p q}^{I}(t) \| f^{I}[t, u, x]\right| d u
$$$$
+\int_{0}^{+\infty}\left|K_{p q}(u)\left\|x_{p q}^{J}(t)\right\| f^{J}[t, u, x]\right| d u
$$$$
\left.+\int_{0}^{+\infty}\left|K_{p q}(u)\right|\left|x_{p q}^{K}(t)\right|\left|f^{K}[t, u, x]\right| d u\right) d t
$$$$
+\int_{0}^{\omega}\left|T_{p q}^{R}(t)\right| d t+\sum_{h=1}^{2 q}\left|I_{p q h}^{R}\left(x_{p q}\left(t_{h}\right)\right)\right|
$$$$
\leq a^{+} \omega\left|x_{p q}^{R}\right|_{\infty}+\int_{0}^{\omega} \sum_{C_{k l} \in N_{r}(p, q)} \bar{C}_{p q}^{k l}
$$$$
\left(\int_{0}^{+\infty}\left|K_{p q}(u)\right| d u\|x\|_{X} M_{f}^{R}\right.
$$$$
+\int_{0}^{+\infty} \mid K_{p q}(u) d u\|x\|_{X} M_{f}^{I}
$$$$
+\int_{0}^{+\infty}\left|K_{p q}(u)\right| d u\|x\|_{X} M_{f}^{J}
$$$$
\left.+\int_{0}^{+\infty}\left|K_{p q}(u)\right| d u\|x\|_{X} M_{f}^{K}\right) d t
$$$$
+\int_{0}^{\omega}\left|T_{p q}^{R}(t)\right| d t+\sum_{h=1}^{2 q}\left|I_{p q h}^{R}\left(x_{p q}\left(t_{h}\right)\right)\right|
$$$$
\leq a^{+} \omega\left|x_{p q}^{R}\right|_{\infty}+\omega\left(M_{f}^{R}+M_{f}^{I}+M_{f}^{J}+M_{f}^{K}\right)
$$$$
\|x\|_{X} \sum_{C_{k l} \in N_{r}(p, q)} \bar{C}_{p q}^{k l} \int_{0}^{+\infty}\left|K_{p q}(u)\right| d u+\omega T
$$$$
+2 q I, \quad p q \in \mathscr{B} \text {. }
$$

Repeating the above procession, for $v=I, J, K$, we can obtain that
Integrating both sides of (29) over the interval $[0, \omega]$, we can obtain

$$
\int_{0}^{\omega} F_{p q v}(t, x) d t+\sum_{h=1}^{2 q} I_{p q h}\left(x_{p q h}\left(t_{h}\right)\right)=0, \quad p q \in \mathscr{B}, v \in E .
$$

\section{Hence,}

$$
\begin{aligned}
& \left|\int_{0}^{\omega} a_{p q}(t) x_{p q}^{R}(t) d t\right| \\
& =\mid \int_{0}^{\omega}\left[\sum _ { C _ { k l } \in N _ { r } ( p , q ) } C _ { p q } ^ { k l } ( t ) \left(\int_{0}^{+\infty} K_{p q}(u) f^{R}[t, u, x] d u x_{p q}^{R}(t)\right.\right. \\
& -\int_{0}^{+\infty} K_{p q}(u) f^{I}[t, u, x] d u x_{p q}^{I}(t) \\
& -\int_{0}^{+\infty} K_{p q}(u) f^{J}[t, u, x] d u x_{p q}^{J}(t) \\
& \left.-\int_{0}^{+\infty} K_{p q}(u) f^{K}[t, u, x] d u x_{p q}^{K}(t)\right) \\
& \left.+T_{p q}^{R}(t)\right] d t+\sum_{h=1}^{2 q} I_{p q h}^{R}\left(x_{p q}\left(t_{h}\right)\right) \\
& \leq \int_{0}^{\omega} \mid \sum_{C_{k l} \in N_{r}(p, q)} C_{p q}^{k l}(t)\left(\int_{0}^{+\infty} K_{p q}(u) f^{R}[t, u, x] d u x_{p q}^{R}(t)\right. \\
& -\int_{0}^{+\infty} K_{p q}(u) f^{I}[t, u, x] d u x_{p q}^{I}(t) \\
& -\int_{0}^{+\infty} K_{p q}(u) f^{J}[t, u, x] d u x_{p q}^{J}(t) \mid \\
& \left.-\int_{0}^{+\infty} K_{p q}(u) f^{K}[t, u, x] d u x_{p q}^{K}(t)\right) \mid d t \\
& +\int_{0}^{\omega}\left|T_{p q}^{R}(t)\right| d t+\sum_{h=1}^{2 q}\left|I_{p q h}^{R}\left(x_{p q}\left(t_{h}\right)\right)\right| \\
& \leq \int_{0}^{\omega} \sum_{C_{k l} \in N_{r}(p, q)} \bar{C}_{p q}^{k l}\left(\int _ { 0 } ^ { + \infty } | K _ { p q } ( u ) | d u \left(M_{f}^{R}+M_{f}^{I}+M_{f}^{J}\right.\right. \\
& \left.\left.+M_{f}^{K}\right)\|x\|_{X}\right) d t+\int_{0}^{\omega}\left|T_{p q}^{R}(t)\right| d t+\sum_{h=1}^{2 q}\left|I_{p q h}^{R}\left(x_{p q}\left(t_{h}\right)\right)\right| \\
& \leq \omega\left(M_{f}^{R}+M_{f}^{I}+M_{f}^{J}+M_{f}^{K}\right)\|x\|_{X} \sum_{C_{k l} \in N_{r}(p, q)} \\
& \cdot \bar{C}_{p q}^{k l} \int_{0}^{+\infty}\left|K_{p q}(u)\right| d u+\omega T+2 q I, \quad p q \in \mathscr{B} .
\end{aligned}
$$


Repeating the above procession, for $v=I, J, K$, we have

$$
\begin{aligned}
\left|\int_{0}^{\omega} a_{p q}(t) x_{p q}^{v}(t) d t\right| \leq & \omega\left(M_{f}^{R}+M_{f}^{I}+M_{f}^{I}+M_{f}^{K}\right) \\
& \cdot\|x\|_{X} \sum_{C_{k l} \in N_{r}(p, q)} \bar{C}_{p q}^{k l} \int_{0}^{+\infty}\left|K_{p q}(u)\right| d u \\
& +\omega T+2 q I, \quad p q \in \mathscr{B} .
\end{aligned}
$$

Since for any $t_{1}, t_{2} \in[0, \omega]$, and $p q \in \mathscr{B}$, we have

$$
\begin{aligned}
& x_{p q}^{v}(t) \leq x_{p q}^{v}\left(t_{1}\right)+\int_{0}^{\omega}\left|\dot{x}_{p q}^{v}(t)\right| d t, \\
& x_{p q}^{v}(t) \geq x_{p q}^{v}\left(t_{2}\right)-\int_{0}^{\omega}\left|\dot{x}_{p q}^{v}(t)\right| d t,
\end{aligned}
$$

then for any $\xi_{p q}^{v}, \eta_{p q}^{v} \in[0, \omega], p q \in \mathscr{B}$, and $v \in E$, we obtain

$$
\begin{aligned}
\int_{0}^{\omega} a_{p q}(t) x_{p q}^{v}(t) d t \leq & \int_{0}^{\omega} a_{p q}(t) x_{p q}^{v}\left(\xi_{p q}^{v}\right) d t \\
& +\int_{0}^{\omega} a_{p q}(t)\left(\int_{0}^{\omega}\left|\dot{x}_{p q}^{v}(t)\right| d t\right) d t, \\
\int_{0}^{\omega} a_{p q}(t) x_{p q}^{v}(t) d t \geq & \int_{0}^{\omega} a_{p q}(t) x_{p q}^{v}\left(\eta_{p q}^{v}\right) d t \\
& -\int_{0}^{\omega} a_{p q}(t)\left(\int_{0}^{\omega}\left|\dot{x}_{p q}^{v}(t)\right| d t\right) d t .
\end{aligned}
$$

Dividing by $\int_{0}^{\omega} a_{p q}(t) d t$ on the both sides of (36) and (37), respectively, we have

$$
\begin{aligned}
x_{p q}^{v}\left(\xi_{p q}^{v}\right) \geq & \frac{1}{\int_{0}^{\omega} a_{p q}(t) d t} \int_{0}^{\omega} a_{p q}(t) x_{p q}^{v}(t) d t \\
& -\int_{0}^{\omega}\left|\dot{x}_{p q}^{v}(t)\right| d t, \quad p q \in \mathscr{B}, v \in E, \\
x_{p q}^{v}\left(\eta_{p q}^{v}\right) \leq & \frac{1}{\int_{0}^{\omega} a_{p q}(t) d t} \int_{0}^{\omega} a_{p q}(t) x_{p q}^{v}(t) d t \\
& +\int_{0}^{\omega}\left|\dot{x}_{p q}^{v}(t)\right| d t, \quad p q \in \mathscr{B}, v \in E .
\end{aligned}
$$

Let $\bar{t}_{p q}^{v}, \underline{t}_{p q}^{v} \in[0, \omega]$ such that $x_{p q}^{v}\left(\bar{t}_{p q}^{v}\right)=\sup _{t \in[0, \omega]} x_{p q}^{v}(t)$, $x_{p q}^{v}\left(\begin{array}{c}t^{\nu} \\ -p q\end{array}\right)=\inf _{t \in[0, \omega]} x_{p q}^{v}(t), p q \in \mathscr{B}$, and $v \in E$; by the arbitrariness of $\xi_{p q}^{v}, \eta_{p q}^{v}$, we can obtain

$$
\begin{aligned}
x_{p q}^{v}\left(\underline{t}_{p q}^{v}\right) \geq & \frac{1}{\int_{0}^{\omega} a_{p q}(t) d t} \int_{0}^{\omega} a_{p q}(t) x_{p q}^{v}(t) d t-\int_{0}^{\omega}\left|\dot{x}_{p q}^{v}(t)\right| d t \\
\geq & -\frac{1}{\int_{0}^{\omega} a_{p q}(t) d t}\left|\int_{0}^{\omega} a_{p q}(t) x_{p q}^{v}(t) d t\right|-\int_{0}^{\omega}\left|\dot{x}_{p q}^{v}(t)\right| d t \\
\geq & -\frac{1}{a^{-} \omega}\left[\omega\left(M_{f}^{R}+M_{f}^{I}+M_{f}^{J}+M_{f}^{K}\right)\|x\|_{X}\right. \\
& \left.\cdot \sum_{C_{k l} \in N_{r}(p, q)} \bar{C}_{p q}^{k l} \int_{0}^{+\infty}\left|K_{p q}(u)\right| d u+\omega T+2 q I\right] \\
& -\left[a^{+} \omega\left|x_{p q}^{v}\right|_{\infty}+\omega\left(M_{f}^{R}+M_{f}^{I}+M_{f}^{J}+M_{f}^{K}\right)\|x\|_{X}\right. \\
& \left.\cdot \sum_{C_{k l} \in N_{r}(i, j)} \bar{C}_{p q}^{k l} \int_{0}^{+\infty}\left|K_{p q}(u)\right| d u+\omega T+2 q I\right] \\
= & -a^{+} \omega\left|x_{p q}^{v}\right|_{\infty}-\left(\frac{1}{a^{-}}+\omega\right) \\
& \cdot\left[\left(M_{f}^{R}+M_{f}^{I}+M_{f}^{J}+M_{f}^{K}\right)\|x\|_{X} \sum_{C_{k l} \in N_{r}(p, q)} \bar{C}_{p q}^{k l}\right. \\
& \left.\times \int_{0}^{+\infty}\left|K_{p q}(u)\right| d u+T+\frac{2 q I}{\omega}\right], \quad p q \in \mathscr{B}, v \in E,
\end{aligned}
$$

and

$$
\begin{aligned}
x_{p q}^{v}\left(\bar{t}_{p q}^{v}\right) \leq & \frac{1}{\int_{0}^{\omega} a_{p q}(t) d t} \int_{0}^{\omega} a_{p q}(t) x_{p q}^{v}(t) d t+\int_{0}^{\omega}\left|\dot{x}_{p q}^{v}(t)\right| d t \\
\leq & \frac{1}{\int_{0}^{\omega} a_{p q}(t) d t} \int_{0}^{\omega}\left|a_{p q}(t) x_{p q}^{v}(t) d t\right|+\int_{0}^{\omega}\left|\dot{x}_{p q}^{v}(t)\right| d t \\
\leq & a^{+} \omega\left|x_{p q}^{v}\right|_{\infty}+\left(\frac{1}{a^{-}}+\omega\right) \\
& \cdot\left[\left(M_{f}^{R}+M_{f}^{I}+M_{f}^{J}+M_{f}^{K}\right)\|x\|_{X} \sum_{C_{k l} \in N_{r}(p, q)} \bar{C}_{p q}^{k l}\right. \\
& \left.\times \int_{0}^{+\infty}\left|K_{p q}(u)\right| d u+T+\frac{2 q I}{\omega}\right], \quad p q \in \mathscr{B}, v \in E .
\end{aligned}
$$

Thus, from (39) and (40) we have

$$
\begin{aligned}
\left|x_{p q}^{v}\right|_{\infty}=\sup _{t \in[0, \omega]}\left|x_{p q}^{v}(t)\right| \leq a^{+} \omega\left|x_{p q}^{v}\right|_{\infty}+\left(\frac{1}{a^{-}}+\omega\right) \\
\cdot\left[\left(M_{f}^{R}+M_{f}^{I}+M_{f}^{J}+M_{f}^{K}\right)\|x\|_{X} \sum_{C_{k l} \in N_{r}(p, q)} \bar{C}_{p q}^{k l}\right. \\
\left.\quad \times \int_{0}^{+\infty}\left|K_{p q}(u)\right| d u+T+\frac{2 q I}{\omega}\right], \quad p q \in \mathscr{B}, v \in E,
\end{aligned}
$$


hence

$$
\|x\|_{X} \leq a^{+} \omega\|x\|_{X}+\left(\frac{1}{a^{-}}+\omega\right)\left[\left(M_{f}^{R}+M_{f}^{I}+M_{f}^{J}+M_{f}^{K}\right)\|x\|_{X} \sum_{C_{k l} \in N_{r}(p, q)} \bar{C}_{p q}^{k l} \times \int_{0}^{+\infty}\left|K_{p q}(u)\right| d u+T+\frac{2 q I}{\omega}\right]
$$

that is

$$
\|x\|_{X} \leq a^{+} \omega\|x\|_{X}+\left(\frac{1}{a^{-}}+\omega\right)\left[\left(M_{f}^{R}+M_{f}^{I}+M_{f}^{J}+M_{f}^{K}\right)\|x\|_{X} \sum_{C_{k l} \in N_{r}(p, q)} \bar{C}_{p q}^{k l} \times \int_{0}^{+\infty}\left|K_{p q}(u)\right| d u+T+\frac{2 q I}{\omega}\right]
$$

Obviously, $A$ is independent of $\lambda$. Take $\Omega=\{x \in X$ : $\left.\|x\|_{X}<A+1\right\}$, then $\Omega \subset X$ with $0 \in \Omega$ such that equation $L u=\lambda N u$ has no solutions in $\partial \Omega \cap \operatorname{Dom} L$ for any $\lambda \in(0$, 1). Thus, by Lemma 1 we conclude that $L x=N x$ has at least one $\omega / 2$-antiperiodic solution in $X$, that is, (16) has at least one $\omega / 2$-antiperiodic solution. By Remark 1, we see that (1) has at least one $\omega / 2$-antiperiodic solution.

\section{The Global Exponential Stability of Antiperiodic Solution}

In this section, by constructing a suitable Lyapunov function we derive sufficient conditions ensuring the global exponential stability of antiperiodic solutions for (1).

Theorem 2. Let H1-H4 and A1 hold. Furthermore, assume that

A2. The impulsive operators satisfy

$$
I_{p q h}\left(x_{p q}\left(t_{h}\right)\right)=-\gamma_{p q h} x_{p q}\left(t_{h}\right), \quad 0 \leq \gamma_{p q h} \leq 2, p q \in \mathscr{B}, h \in \mathbb{N}
$$

A3. There exists a positive constant $\lambda>0$ satisfying

$$
\delta=\max _{p q \in \mathscr{B}}\left\{\delta_{p q}\right\}<0,
$$

where

$$
\begin{aligned}
\delta_{p q}= & \left(\lambda-a^{-}\right)+\sum_{C_{k l} \in N_{r}(p, q)} \bar{C}_{p q}^{k l} \int_{0}^{+\infty}\left|K_{p q}(u)\right| \\
\cdot & {\left[\left(M_{f}^{R}+M_{f}^{I}+M_{f}^{J}+M_{f}^{K}\right)\right.} \\
& \left.+4\left(L_{f}^{R}+L_{f}^{I}+L_{f}^{J}+L_{f}^{K}\right)\right] d u,
\end{aligned}
$$

then, the $\omega / 2$-antiperiodic solution of (1) is globally exponentially stable.

Proof 2. By Theorem 1, (16) has an $\omega / 2$-periodic solution. Let $x(t)$ be the $\omega / 2$-periodic solution with the initial value $\varphi(t)$ and $y(t)$ be an arbitrary solution with the initial value $\psi(t)$. Set $z_{p q}^{v}(t)=x_{p q}^{v}(t)-y_{p q}^{v}(t), p q \in \mathscr{B}$, and $v \in E$. By (16), for $t>0$ and $t \neq t_{h}$, we have

$$
\begin{aligned}
D^{+}\left|z_{p q}^{v}(t)\right| \leq & -a^{-}\left|z_{p q}^{v}(t)\right|+\sum_{C_{k l} \in N_{r}(p, q)} \bar{C}_{p q}^{k l} \int_{0}^{+\infty} K_{p q}(u) \\
& \cdot\left[M_{f}^{R}\left|z_{p q}^{R}(t)\right|+M_{f}^{I}\left|z_{p q}^{I}(t)\right|+M_{f}^{J}\left|z_{p q}^{J}(t)\right|\right. \\
& +M_{f}^{K}\left|z_{p q}^{K}(t)\right|+4\left(L_{f}^{R}\left|z_{k l}^{R}(t-u)\right|\right. \\
& +L_{f}^{I}\left|z_{k l}^{I}(t-u)\right|+L_{f}^{J}\left|z_{k l}^{J}(t-u)\right| \\
& \left.\left.+L_{f}^{K}\left|z_{k l}^{K}(t-u)\right|\right)\right] d u, \quad p q \in \mathscr{B}, v \in E .
\end{aligned}
$$

For $t=t_{h}, h \in \mathbb{N}$, from A2 we can have the following:

$$
\begin{aligned}
\left|x_{p q}^{v}\left(t_{h}^{+}\right)-y_{p q}^{v}\left(t_{h}^{+}\right)\right| & =\left|1-\gamma_{p q h}\right|\left|x_{p q}^{v}\left(t_{h}\right)-y_{p q}^{v}\left(t_{h}\right)\right| \\
& \leq\left|x_{p q}^{v}\left(t_{h}\right)-y_{p q}^{v}\left(t_{h}\right)\right|,
\end{aligned}
$$

that is

$$
\left|z_{p q}^{v}\left(t_{h}^{+}\right)\right| \leq\left|z_{p q}^{v}\left(t_{h}\right)\right|
$$

where $p q \in \mathscr{B}$ and $v \in E$.

Construct the Lyapunov function $V(t)$ as follows:

$$
V(t)=V^{R}(t)+V^{I}(t)+V^{J}(t)+V^{K}(t)
$$

where $V^{v}(t)=\sum_{p q \in \mathscr{B}}\left|z_{p q}^{v}(t)(t)\right| e^{\lambda t}, p q \in \mathscr{B}$, and $v \in E$. 

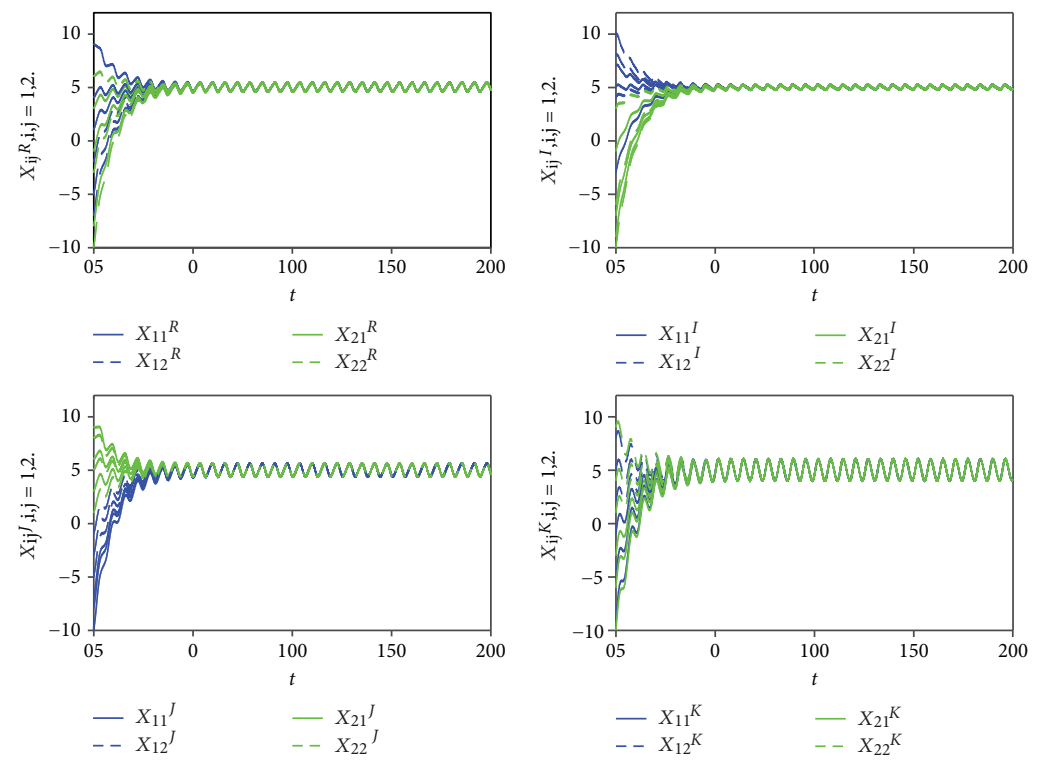

FIGURE 1: The states of four parts of $x_{11}, x_{12}, x_{21}$, and $x_{22}$.

Calculating the upper right derivative of $V^{R}(t)$ along the solutions of (16), for $t \neq t_{h}$, we obtain

$$
\begin{aligned}
D^{+} V^{R}(t)= & \sum_{p q \in \mathscr{B}}\left\{\lambda e^{\lambda t}\left|z_{p q}^{R}(t)\right|+\operatorname{sign}\left(z_{p q}^{R}(t)\right) D^{+}\left(z_{p q}^{R}(t)\right) e^{\lambda t}\right\} \\
\leq & \sum_{p q \in \mathscr{B}}\left\{\left(\lambda-a^{-}\right) e^{\lambda t}\left|z_{p q}^{R}\right|+e^{\lambda t} \sum_{C_{k l} \in N_{r}(p, q)}\right. \\
& \cdot \bar{C}_{p q}^{k l} \int_{0}^{+\infty} K_{p q}(u)\left[\left(M_{f}^{R}\left|z_{p q}^{R}(t)\right|+M_{f}^{I}\left|z_{p q}^{I}(t)\right|\right.\right. \\
& \left.+M_{f}^{J}\left|z_{p q}^{J}(t)\right|+M_{f}^{K}\left|z_{p q}^{K}(t)\right|\right)+4\left(L_{f}^{R}\left|z_{k l}^{R}(t-u)\right|\right. \\
& \left.\left.+L_{f}^{I}\left|z_{k l}^{I}(t-u)\right|+L_{f}^{J}\left|z_{k l}^{J}(t-u)\right|\right] d u\right\} \\
\leq & e^{\lambda t} \sum_{p q \in \mathscr{B}}\left\{\left(\lambda-a^{-}\right)+\sum_{C_{k l} \in N_{r}(p, q)} \bar{C}_{p q}^{k l} \int_{0}^{+\infty}\left|K_{p q}(u)\right|\right. \\
& \cdot\left[\left(M_{f}^{R}+M_{f}^{I}+M_{f}^{J}+M_{f}^{K}\right)\right. \\
& \left.\left.+4\left(L_{f}^{R}+L_{f}^{I}+L_{f}^{J}+L_{f}^{K}\right)\right] d u\right\}\|z\| .
\end{aligned}
$$

Repeat the same calculation and we can get

$$
\begin{aligned}
D^{+} V^{v}(t) \leq & e^{\lambda t} \sum_{p q \in \mathscr{B}}\left\{\left(\lambda-a^{-}\right)+\sum_{C_{k l} \in N_{r}(p, q)} \bar{C}_{p q}^{k l} \int_{0}^{+\infty}\left|K_{p q}(u)\right|\right. \\
\cdot & {\left[\left(M_{f}^{R}+M_{f}^{I}+M_{f}^{J}+M_{f}^{K}\right)\right.} \\
+ & \left.\left.4\left(L_{f}^{R}+L_{f}^{I}+L_{f}^{J}+L_{f}^{K}\right)\right] d u\right\}\|z\|, \quad v \in E, t \neq t_{h} .
\end{aligned}
$$

It follows from A3, (51), and (52) that for $t \neq t_{h}$,

$$
D^{+} V(t) \leq 0
$$

By (49), we also have

$$
\begin{aligned}
V\left(t_{h}^{+}\right)= & V^{R}\left(t_{h}^{+}\right)+V^{I}\left(t_{h}^{+}\right)+V^{J}\left(t_{h}^{+}\right)+V^{K}\left(t_{h}^{+}\right) \\
= & \sum_{p q \in \mathscr{B}}\left|z_{p q}^{R}\left(t_{h}^{+}\right)\right| e^{\lambda t_{h}^{+}}+\sum_{p q \in \mathscr{B}}\left|z_{p q}^{I}\left(t_{h}^{+}\right)\right| e^{\lambda t_{h}^{+}} \\
& +\sum_{p q \in \mathscr{B}}\left|z_{p q}^{J}\left(t_{h}^{+}\right)\right| e^{\lambda t_{h}^{+}}+\sum_{p q \in \mathscr{B}}\left|z_{p q}^{K}\left(t_{h}^{+}\right)\right| e^{\lambda t_{h}^{+}} \\
\leq & \sum_{p q \in \mathscr{B}}\left|z_{p q}^{R}\left(t_{h}\right)\right| e^{\lambda t_{h}}+\sum_{p q \in \mathscr{B}}\left|z_{p q}^{I}\left(t_{h}\right)\right| e^{\lambda t_{h}} \\
& +\sum_{p q \in \mathscr{B}}\left|z_{p q}^{J}\left(t_{h}\right)\right| e^{\lambda t_{h}}+\sum_{p q \in \mathscr{B}}\left|z_{p q}^{K}\left(t_{h}\right)\right| e^{\lambda t_{h}} \\
= & V\left(t_{h}\right), \quad p q \in \mathscr{B}, h \in \mathbb{N} .
\end{aligned}
$$

Hence, $V(t) \leq V(0)$ for all $t \geq 0$.

On the other hand, we have

$$
\begin{aligned}
V(0)= & V^{R}(0)+V^{I}(0)+V^{J}(0)+V^{K}(0) \\
= & \sum_{p q \in \mathscr{B}}\left(\left|z_{p q}^{R}(0)\right|+\left|z_{p q}^{I}(0)\right|+\left|z_{p q}^{J}(0)\right|+\left|z_{p q}^{K}(0)\right|\right) \\
\leq & \sum_{p q \in \mathscr{B}}\left\{\sup _{s \in(-\infty, 0]}\left(\left|z_{p q}^{R}(s)\right|+\left|z_{p q}^{I}(s)\right|+\left|z_{p q}^{J}(s)\right|+\left|z_{p q}^{K}(s)\right|\right)\right\} \\
\leq & \sum_{p q \in \mathscr{B}}\left\{\operatorname { s u p } _ { s \in ( - \infty , 0 ] } \left(\left|\varphi_{p q}^{R}(s)-\psi_{p q}^{R}(s)\right|+\left|\varphi_{p q}^{I}(s)-\psi_{p q}^{I}(s)\right|\right.\right. \\
& \left.\left.+\left|\varphi_{p q}^{J}(s)-\psi_{p q}^{J}(s)\right|+\left|\varphi_{p q}^{K}(s)-\psi_{p q}^{K}(s)\right|\right)\right\}=\|\varphi-\psi\| .
\end{aligned}
$$



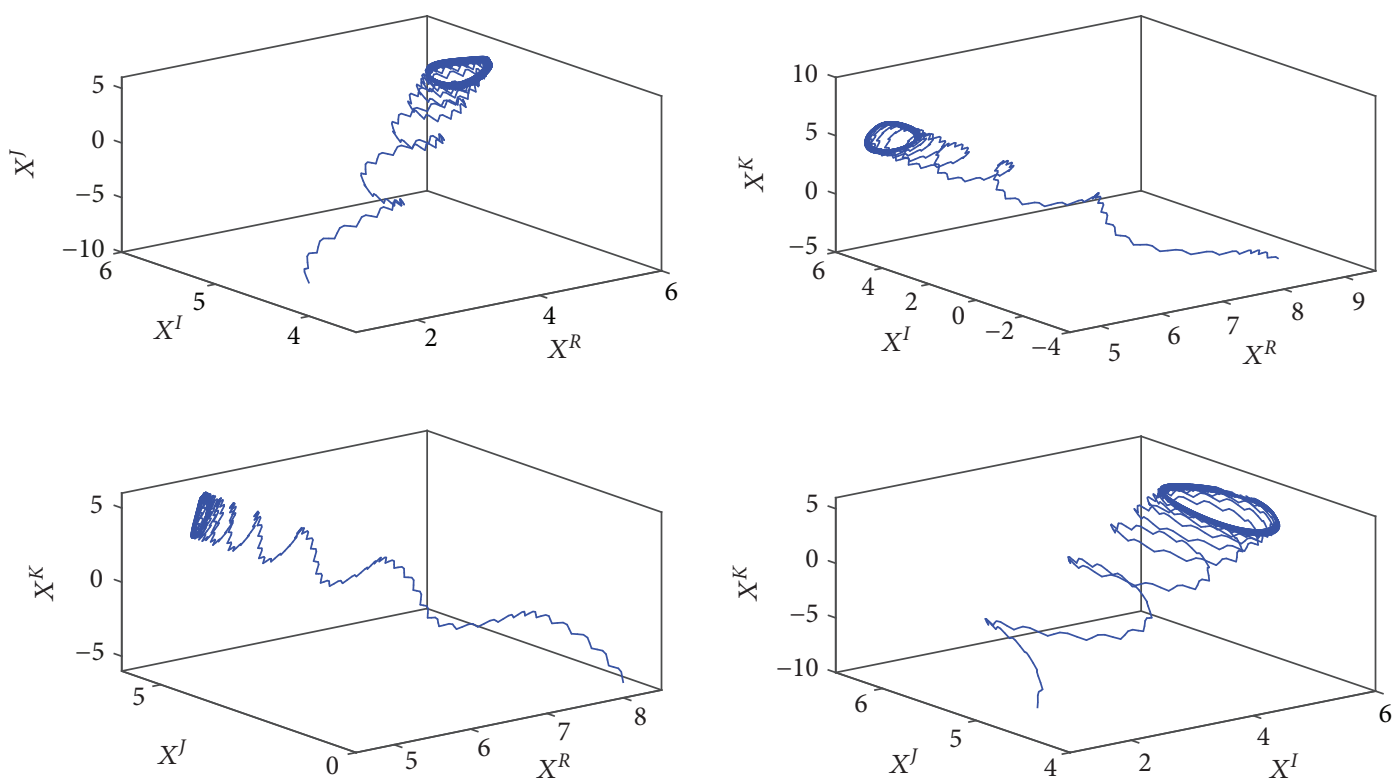

Figure 2: Curves of $x$ in 3-dimensional space for stable case.

Let $M=1$, and we can easily obtain the following:

$\|x(t)-y(t)\|_{0} \leq V(t) e^{-\lambda t} \leq V(t) e^{-\lambda t} \leq M\|\varphi-\psi\| e^{-\lambda t}, \quad t \geq 0$.

Therefore, the $\omega / 2$-antiperiodic solution of (16) is globally exponentially stable. According to Remark 1, we know that the $\omega / 2$-antiperiodic solution of (16) is globally exponentially stable. The proof is complete.

\section{An Illustrative Example}

In this section, we give an example to show the feasibility and effectiveness of the results obtained in this paper.

Example 1. Consider the following QVSICNN:

$$
\left\{\begin{array}{l}
\dot{x}_{p q}(t)=-a_{p q}(t) x_{p q}(t)-\sum_{C_{k l} \in N_{r}(p, q)} C_{p q}^{k l}(t) \int_{0}^{+\infty} K_{p q}(u) x_{p q}(t) f\left(x_{k l}(t-u)\right) d u+T_{p q}(t), \quad t \geq 0, t \neq t_{h}, \\
\Delta x_{p q}\left(t_{h}\right)=x_{p q}\left(t_{h}^{+}\right)-x_{p q}\left(t_{h}^{-}\right)=I_{p q h}\left(x_{p q}\left(t_{h}\right)\right), \quad t=t_{h},
\end{array}\right.
$$

where $\dot{x}_{p q}(t)=\dot{x}_{p q}^{R}(t)+i \dot{x}_{p q}^{I}(t)+j \dot{x}_{p q}^{I}(t)+k \dot{x}_{p q}^{K}(t) \in \mathbb{Q}, \quad K_{p q}$ $(u)=e^{-u}, p, q=1,2$, and the coefficients are as follows:

$$
\begin{aligned}
f(x)= & 0.01\left|\sin x^{R}\right|+i 0.02\left|\sin x^{I}\right| \\
& +j 0.03\left|\sin x^{J}\right|+k 0.04\left|\sin x^{K}\right|, \\
a_{11}(t)= & a_{12}(t)=a_{21}(t)=a_{22}(t)=\frac{1}{8 \pi} \sin ^{2} t+\frac{1}{4 \pi}, \\
C_{11}(t)= & C_{12}(t)=C_{21}(t)=C_{22}(t)=0.01 \cos ^{2} t, \\
T_{11}(t)= & T_{12}(t)=T_{21}(t)=T_{22}(t) \\
= & 0.2 \sin t+i 0.1 \cos t+j 0.3 \sin t \\
& +k 0.5 \cos t,
\end{aligned}
$$

and the impulsive functions $I_{p q h}\left(x_{p q}\left(t_{h}\right)\right)=-0.001 x_{p q}\left(t_{h}\right)$, $p, q=1,2, t_{h}=h \omega / 2$, and $h \in \mathbb{N}$.
By a simple calculation, we can get

$$
\begin{aligned}
a^{-} & =\frac{1}{4 \pi}, \\
a^{+} & =\frac{1}{8 \pi}+\frac{1}{4 \pi}, \\
\omega & =2 \pi, \\
\gamma_{p q h} & =0.001, \\
I & =0.001, \\
\int_{0}^{+\infty} K_{p q}(u) d u & =1,
\end{aligned}
$$

$$
M_{f}^{R}=0.01,
$$




$$
\begin{aligned}
M_{f}^{I} & =0.02, \\
M_{f}^{J} & =0.03, \\
M_{f}^{K}= & 0.04, \\
L_{f}^{R}= & L_{f}^{I}=L_{f}^{J}=L_{f}^{K}=0.04, \\
\sum_{C_{k l} \in N_{1}(1,1)} \bar{C}_{11}^{k l}= & \sum_{C_{k l} \in N_{1}(1,2)} \bar{C}_{12}^{k l}=\sum_{C_{k l} \in N_{1}(2,1)} \bar{C}_{21}^{k l} \\
= & \sum_{C_{k l} \in N_{1}(2,2)} \bar{C}_{22}^{k l}=0.04, \\
\Gamma= & \frac{a^{-} \omega\left(1-a^{+} \omega\right)}{1+a^{-} \omega}-\left(M_{f}^{R}+M_{f}^{I}+M_{f}^{J}+M_{f}^{K}\right) \\
& \cdot \sum_{C_{k l} \in N_{r}(p, q)} \bar{C}_{p q}^{k l} \int_{0}^{+\infty}\left|K_{p q}(u)\right| d u \approx 0.08>0 .
\end{aligned}
$$

Taking $\lambda=0.01$, we obtain

$$
\begin{aligned}
\max _{1 \leq i, j \leq 2}\left\{\delta_{p q}\right\}= & \left(\lambda-a^{-}\right)+\sum_{C_{k l} \in N_{r}(p, q)} \bar{C}_{p q}^{k l} \int_{0}^{+\infty}\left|K_{p q}(u)\right| \\
& \cdot\left[\left(M_{f}^{R}+M_{f}^{I}+M_{f}^{J}+M_{f}^{K}\right)\right. \\
& \left.+4\left(L_{f}^{R}+L_{f}^{I}+L_{f}^{J}+L_{f}^{K}\right)\right] \mathrm{d} u \approx-0.04<0 .
\end{aligned}
$$

Thus conditions $\mathrm{H} 1-\mathrm{H} 4$ and $\mathrm{A} 1-\mathrm{A} 3$ hold. Therefore, according to Theorems 1 and 2, (57) has at least one $\pi$-antiperiodic solution, which is globally exponentially stable (see Figures 1 and 2).

\section{Conclusion}

In this paper, we investigated the existence and global exponential stability of antiperiodic solutions for a class of QVSICNNs with impulsive effects. We introduce a new method different from all other antiperiodic solutions of neural networks in a previous work. By using a new continuation theorem of the coincidence degree theory and constructing a suitable Lyapunov function, we obtain the existence and global exponential stability results for an antiperiodic solution. However, in this paper, we only investigate the antiperiodic solution problem of QVSICNNs with impulsive effects. In future work, periodic solution, almost periodic solution, and pseudo almost periodic solution in the quaternion field can be considered.

\section{Data Availability}

No data were used to support this study.

\section{Conflicts of Interest}

The authors declare that they have no conflict of interest.

\section{Acknowledgments}

This work is supported by the National Natural Science Foundation of the People's Republic of China under Grant nos. 11861072 and 11361072 .

\section{References}

[1] A. Bouzerdoum, B. Nabet, and R. B. Pinter, "Analysis and analog implementation of directionally sensitive shunting inhibitory neural networks," Visual Information Processing: From Neurons to Chips, vol. 1473, pp. 29-38, 1991, SPIE.

[2] A. Bouzerdoum and R. B. Pinter, "Nonlinear lateral inhibition applied to motion detection in the fly visual system," in Nonlinear Vis, R. B. Pinter and B. Nabet, Eds., pp. 423-450, CRC Press, Boca Raton, FL, 1992.

[3] R. Yang, B. Wu, and Y. Liu, "A Halanay-type inequality approach to the stability analysis of discrete-time neural networks with delays," Applied Mathematics and Computation, vol. 265, pp. 696-707, 2015.

[4] Y. Li, C. Liu, and L. Zhu, "Global exponential stability of periodic solution for shunting inhibitory CNNs with delays," Physics Letters A, vol. 337, no. 1-2, pp. 46-54, 2005.

[5] Y. Li and C. Wang, "Almost periodic solutions of shunting inhibitory cellular neural networks on time scales," Communications in Nonlinear Science and Numerical Simulation, vol. 17, no. 8, pp. 3258-3266, 2012.

[6] C. Xu and P. Li, "On anti-periodic solutions for neutral shunting inhibitory cellular neural networks with time-varying delays and D operator," Neurocomputing, vol. 275, pp. 377382, 2018.

[7] M. U. Akhmet and M. O. Fen, "Attraction of Li-Yorke chaos by retarded SICNNs," Neurocomputing, vol. 147, pp. 330342, 2015.

[8] B. Liu, "Global exponential convergence of non-autonomous SICNNs with multi-proportional delays," Neural Computing and Applications, vol. 28, no. 8, pp. 1927-1931, 2017.

[9] A. Zhang, "Pseudo almost periodic solutions for SICNNs with oscillating leakage coefficients and complex deviating arguments," Neural Processing Letters, vol. 45, no. 1, pp. 183-196, 2017.

[10] A. Zhang, "Almost periodic solutions for SICNNs with neutral type proportional delays and D operators," Neural Processing Letters, vol. 47, no. 1, pp. 57-70, 2018.

[11] Y. Liu, H. Chen, and B. Wu, "Controllability of Boolean control networks with impulsive effects and forbidden states," Mathematical Methods in the Applied Sciences, vol. 37, no. 1, pp. 1-9, 2014.

[12] M. Şaylı and E. Yılmaz, "Periodic solution for state-dependent impulsive shunting inhibitory CNNs with time-varying delays," Neural Networks, vol. 68, pp. 1-11, 2015.

[13] Y. Li and J. Shu, "Anti-periodic solutions to impulsive shunting inhibitory cellular neural networks with distributed delays on time scales," Communications in Nonlinear Science and Numerical Simulation, vol. 16, no. 8, pp. 33263336, 2011.

[14] T. Isokawa, N. Matsui, and H. Nishimura, "Quaternionic neural networks: fundamental properties and applications," in Complex Valued Neural Networks: Utilizing HighDimensional Parameters, pp. 411-439, IGI Global, 2009. 
[15] N. Matsui, T. Isokawa, H. Kusamichi, F. Peper, and H. Nishimura, "Quaternion neural network with geometrical operators," Journal of Intelligent \& Fuzzy Systems, vol. 15, no. 3-4, pp. 149-164, 2004.

[16] H. Kusamichi, T. Isokawa, N. Matsui, Y. Ogawa, and K. Maeda, "A new scheme for colour night vision by quaternion neural network," in 2nd International Conference on Autonomous Robots and Agents, pp. 101-106, Palmerston North, New Zealand, December 2004.

[17] Y. Li, X. Meng, and Y. Ye, "Almost periodic synchronization for quaternion-valued neural networks with time-varying delays," Complexity, vol. 2018, Article ID 6504590, 13 pages, 2018.

[18] Y. Liu, D. Zhang, and J. Lu, "Global exponential stability for quaternion-valued recurrent neural networks with timevarying delays," Nonlinear Dynamics, vol. 87, no. 1, pp. 553$565,2017$.

[19] D. Zhang, K. I. Kou, Y. Liu, and J. Cao, "Decomposition approach to the stability of recurrent neural networks with asynchronous time delays in quaternion field," Neural Networks, vol. 94, pp. 55-66, 2017.

[20] Y. Li and X. Meng, "Existence and global exponential stability of pseudo almost periodic solutions for neutral type quaternion-valued neural networks with delays in the leakage term on time scales," Complexity, vol. 2017, Article ID 9878369, 15 pages, 2017.

[21] Y. Liu, D. Zhang, J. Lu, and J. Cao, "Global $\mu$-stability criteria for quaternion-valued neural networks with unbounded time-varying delays," Information Sciences, vol. 360, pp. 273288, 2016.

[22] X. You, Q. Song, J. Liang, Y. Liu, and F. E. Alsaadi, "Global $\mu$-stability of quaternion-valued neural networks with mixed time-varying delays," Neurocomputing, vol. 290, pp. 12-25, 2018.

[23] Y. Li and J. Qin, "Existence and global exponential stability of periodic solutions for quaternion-valued cellular neural networks with time-varying delays," Neurocomputing, vol. 292, pp. 91-103, 2018.

[24] C. A. Popa and E. Kaslik, "Multistability and multiperiodicity in impulsive hybrid quaternion-valued neural networks with mixed delays," Neural Networks, vol. 99, pp. 1-18, 2018.

[25] A. Abdurahman and H. Jiang, "The existence and stability of the anti-periodic solution for delayed Cohen-Grossberg neural networks with impulsive effects," Neurocomputing, vol. 149, pp. 22-28, 2015.

[26] Y. Li, L. Yang, and W. Wu, "Anti-periodic solution for impulsive BAM neural networks with time-varying leakage delays on time scales," Neurocomputing, vol. 149, pp. 536$545,2015$.

[27] M. Şaylı and E. Yılmaz, "Anti-periodic solutions for statedependent impulsive recurrent neural networks with timevarying and continuously distributed delays," Annals of Operations Research, vol. 258, no. 1, pp. 159-185, 2017.

[28] Q. Wang, Y. Fang, H. Li, L. Su, and B. Dai, "Anti-periodic solutions for high-order Hopfield neural networks with impulses," Neurocomputing, vol. 138, pp. 339-346, 2014.

[29] Z. Long, "New results on anti-periodic solutions for SICNNs with oscillating coefficients in leakage terms," Neurocomputing, vol. 171, pp. 503-509, 2016.
[30] L. Peng and W. Wang, "Anti-periodic solutions for shunting inhibitory cellular neural networks with time-varying delays in leakage terms," Neurocomputing, vol. 111, pp. 27-33, 2013.

[31] J. Shao, "Anti-periodic solutions for shunting inhibitory cellular neural networks with time-varying delays," Physics Letters A, vol. 372, no. 30, pp. 5011-5016, 2008.

[32] Q. Zhou, “Anti-periodic solutions for cellular neural networks with oscillating coefficients in leakage terms," International Journal of Machine Learning and Cybernetics, vol. 8, no. 5, pp. 1607-1613, 2017.

[33] S. Shen, B. Li, and Y. Li, "Anti-periodic dynamics of quaternion-valued fuzzy cellular neural networks with timevarying delays on time scales," International Journal of Machine Learning and Cybernetics, vol. 2018, pp. 1-14, 2018.

[34] A. Sudbery, "Quaternionic analysis," Mathematical Proceedings of the Cambridge Philosophical Society, vol. 85, no. 2, pp. 199-225, 1979.

[35] P. Amster, Topological Methods in the Study of Boundary Valued Problems, Springer, New York, 2013. 


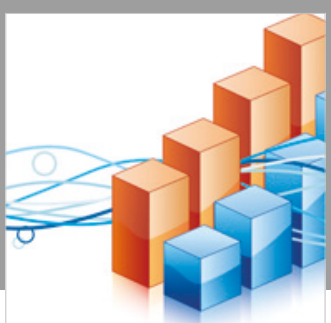

Advances in

Operations Research

\section{-n-m}
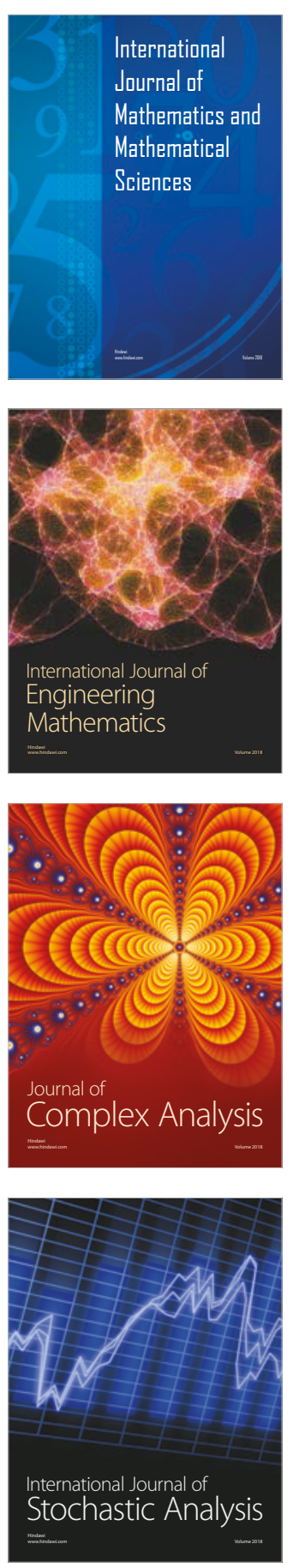
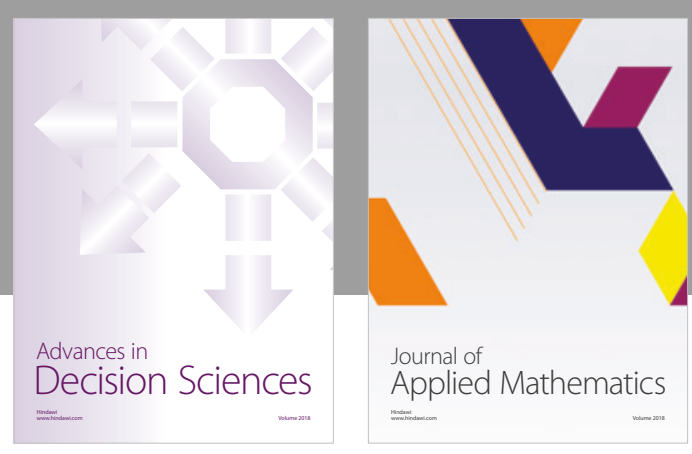

Journal of

Applied Mathematics
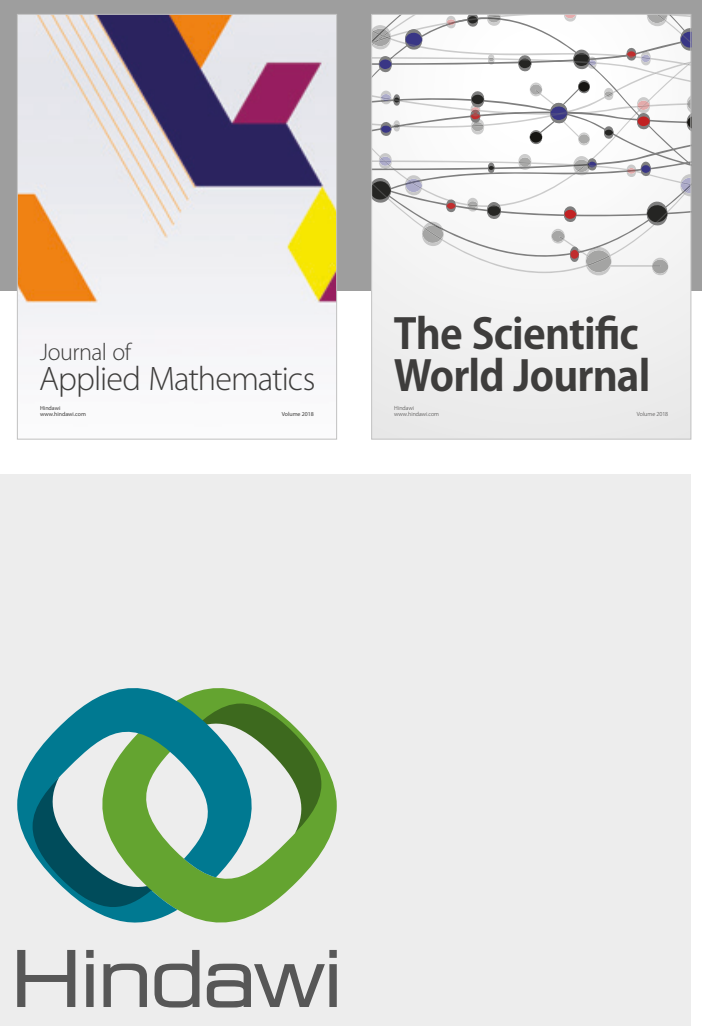

Submit your manuscripts at

www.hindawi.com

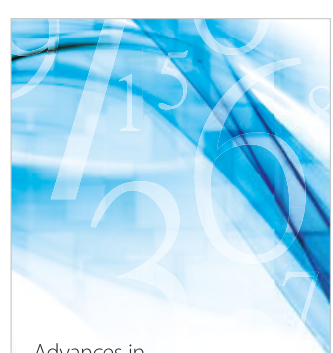

Advances in
Numerical Analysis
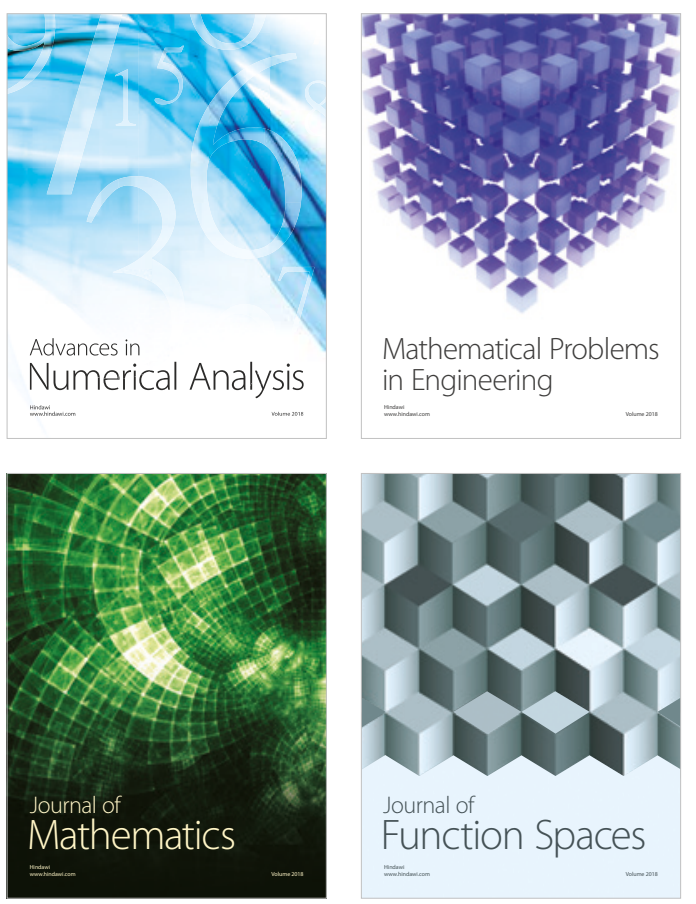

Mathematical Problems in Engineering

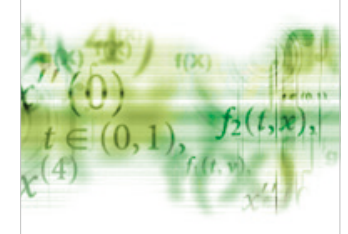

International Journal of

Differential Equations

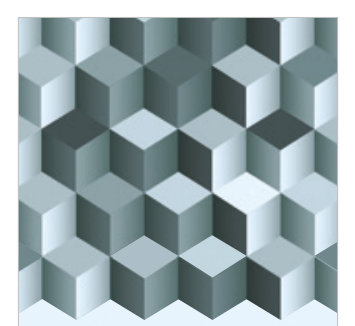

Journal of

Function Spaces
The Scientific

World Journal

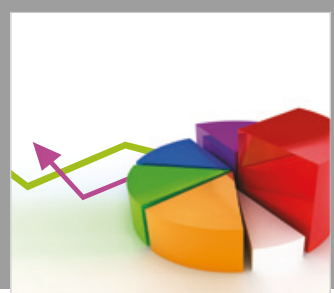

Journal of

Probability and Statistics
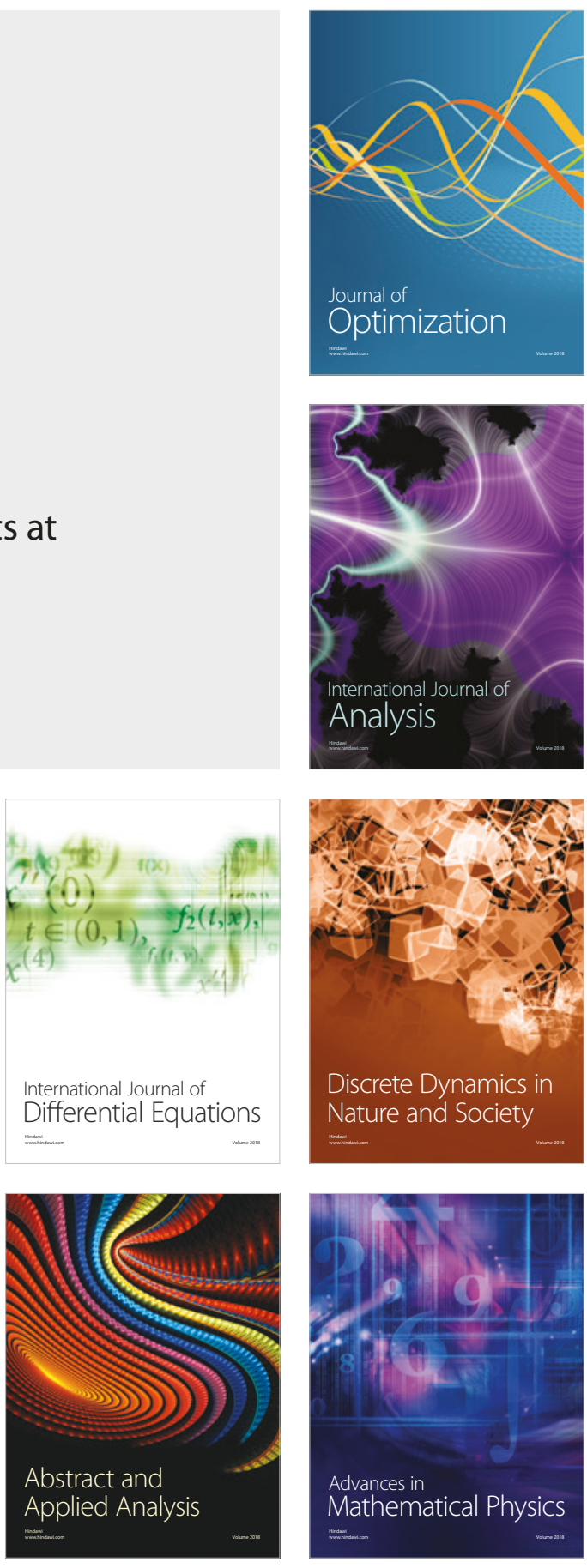